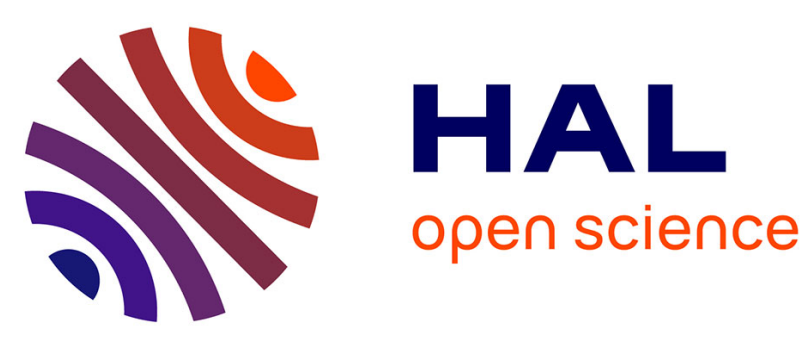

\title{
Modulated Wideband Converter Compressed Sensing Spectrum Reconstruction in Multi-Level Power of Transmitters Signal Scenarios
}

\author{
Lap Luat Nguyen, Anthony Fiche, Roland Gautier
}

\section{To cite this version:}

Lap Luat Nguyen, Anthony Fiche, Roland Gautier. Modulated Wideband Converter Compressed Sensing Spectrum Reconstruction in Multi-Level Power of Transmitters Signal Scenarios. International Conference on Advanced Technologies for Communications (ATC), Oct 2021, Ho Chi Minh City, Vietnam. hal-03379719

\author{
HAL Id: hal-03379719 \\ https://hal.science/hal-03379719
}

Submitted on 19 Oct 2021

HAL is a multi-disciplinary open access archive for the deposit and dissemination of scientific research documents, whether they are published or not. The documents may come from teaching and research institutions in France or abroad, or from public or private research centers.
L'archive ouverte pluridisciplinaire HAL, est destinée au dépôt et à la diffusion de documents scientifiques de niveau recherche, publiés ou non, émanant des établissements d'enseignement et de recherche français ou étrangers, des laboratoires publics ou privés. 


\title{
Modulated Wideband Converter Compressed Sensing Spectrum Reconstruction in Multi-Level Power of Transmitters Signal Scenarios
}

\author{
Lap Luat Nguyen ${ }^{1,2}$, Anthony Fiche ${ }^{3}$, and Roland Gautier ${ }^{3}$ \\ ${ }^{1}$ School of Electrical Engineering, International University, Ho Chi Minh city, Vietnam \\ ${ }^{2}$ Vietnam National University, Ho Chi Minh city, Vietnam \\ ${ }^{3}$ Univ Brest, Lab-STICC, CNRS, UMR 6285, F-29200, Brest, France \\ Email: 1,2 nlluat@hcmiu.edu.vn, and ${ }^{3}\{$ firstname.name\}@univ-brest.fr
}

\begin{abstract}
This paper studies the impacts of transmitters power levels on spectrum reconstruction performances in order to monitor a wideband spectrum. A sub-Nyquist blind sampling scheme based on the Modulated Wideband Converter (MWC) has been considered to achieve this task. Reconstruction performances are evaluated in simulation in terms of correct reconstruction and false alarm rates by studying several multi-level power scenarios. The study shows that the performance of reconstruction depends on the In-Band Signal-to-Noise ratio (IBSNR) of each transmitter. Based on these results, a threshold of In-Band Signal-to-Noise ratio to ensure the correct reconstruction is proposed.
\end{abstract}

\section{INTRODUCTION}

In context of cognitive radio, the limitation of frequency resources motivates the studies on frequency sharing [1], [2]. To not affect the primary (licensed) users, the secondary users must know whether the frequency band is vacant. A very common way to detect the present of power spectrum at licensed band is to apply an energy detector, since it is low complexity, however it is sensitive to noise level [3]. Another possible method is considered as spectrum sensing, which can detect multiple power level of primary users and secondary users [4], [5]. A problem of spectrum sensing that needs to be taken in account is the sensed bandwidth. In some applications, the sensed bandwidth may go beyond several GHz. Consequently, the limited sampling rate and limited input bandwidth of a conventional Analog-to-Digital Converter (ADC) will be a constraint for spectrum sensing. In the literature, the subNyquist sampling methods such as Random Modulator [6], [7], Multicoset Sampling [8], [9] or Modulated Wideband Converter (MWC) [10]-[12] are seen as a premise to deploy spectrum sensing into practice. In [13], a multicoset based on Compressed Sensing (CS) [14], [15] is proposed to estimate the power spectral density, at sub-Nyquist sampled wide sense-stationary. To the best of our knowledge, the MWC seems to be the most convenient scheme in terms of practical implementation, since it has been deployed by different prototypes and analog boards [11], [16], [17].

In practice, primary users use multiband spectra with different parameters of interest, such as power, bandwidth or modulation. The MWC has potential of wideband spectrum sensing and multiband detection in real-time, which benefits on practical implementation than other schemes. As a result, it comes up with some practical applications in cognitive radio [18], [19], and fast spectrum sensing and receiver flexibility in spectrum sensing applications [20] and meet the LTE specifications [21].

The MWC has proved its efficiency to monitor multiband spectra, such as Phase Shift Keying modulation scenario [16] or rectangular shapes [10] with different energy ratios. However, the authors do not focus on the detection sensibility of transmitter with low power level. For example, in cognitive radio, the low power signal detection could bring benefit. In case of frequency sharing, an idle band can be occupied by a secondary user with very weak signal strength, to without affects the primary user. A spectrum sensing system can verify the presence of the secondary user. The attempt to detect very low power level transmitter contributes to the study of fading for both primary and secondary users [22], [23]. To balance the fading in cognitive radio, the primary user can increase its performance if the channel information is known, especially the SNR. Consequently, the motivation of this paper is to study the impacts of amplitude level ratio between transmitters on spectrum reconstruction performances (correct reconstruction and false alarm rates) and propose a threshold based on our simulations which ensure high reconstruction performances.

This paper is organized as follows. Section II presents the background of the MWC. In Section III, several multi-level power scenarios are studied by comparing In-Band Signal-toNoise Ratio of all transmitters (called global in-Band SNR and noticed IBSNR $_{\mathrm{G}}$ ) and In-Band SNR of each transmitter taken separately (noticed $\mathrm{IBSNR}_{1 \mathrm{~T}}$ ). Then, the evaluation of the simulation results based on the correct reconstruction rate and false alarm rate according to the proposed scenarios is presented.

\section{Modulated Wideband CONVERTER}

\section{A. Physical scheme and operation description}

The MWC physical scheme [12] is shown in Fig. 1. A wideband signal $x(t)$ comprises multiband, but sparse and unknown carrier frequency. The signal $x(t)$ is considered as 
an input of the MWC scheme. This scheme consists of $M$ physical channels, each has one mixer, one lowpass filter and one ADC. The mixer with mixing sequence $p_{i}(t)$ is to shift all spectra of $x(t)$ into baseband. The mixing sequence $p_{i}(t)$ can be any sequence in communications such as Gold [24], Kasami [25] or random sequences with the repetition frequency $F_{p}$. The lowpass filter keeps the signal only in baseband bandwidth $\left[-F_{s} / 2 ; F_{s} / 2\right]$, with cutoff frequency $F_{c}=F_{s} / 2$. Then, the sampling rate of ADC will be chosen $F_{s}$ for convenience.

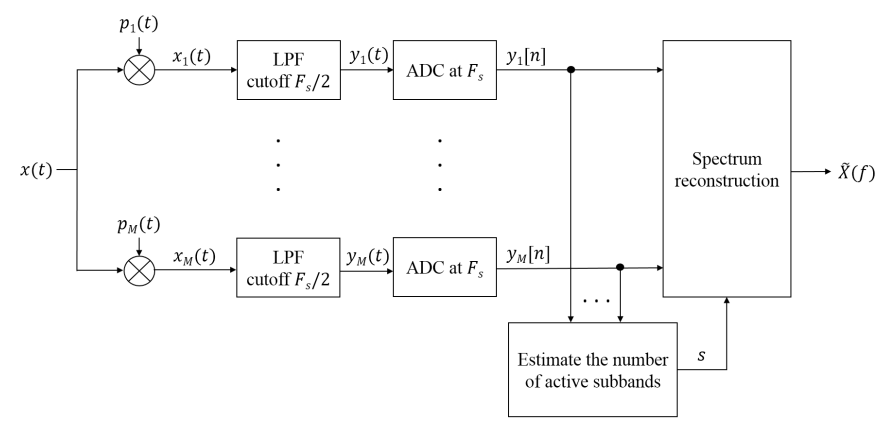

Fig. 1: The MWC scheme and processing stages.

The output of the mixer is

$$
x_{i}(t)=p_{i}(t) x(t)
$$

and the output of lowpass filter $y_{i}(t)$ as

$$
y_{i}(t)=h(t) * x_{i}(t),
$$

where $h(t)$ the ideal lowpass filter transfer function. At last, the conversion output reads as

$$
y_{i}[n]=y_{i}\left(n T_{s}\right),
$$

where $T_{s}$ the sampling period. For further development, consider that the Nyquist bandwidth is divided into $L$ subbands, then the relationship between Nyquist frequency and mixing frequency is $L=F_{N y q} / F_{p}$. It means that each subband has $F_{p}$ bandwidth. The active subband $l$ which comprises the input spectrum will be detected among $L$ subbands ( $L=2 L_{0}+1$ and $\left.-L_{0} \leq l \leq L_{0}\right)$ by the MWC scheme and Compressed Sensing technique [14], [15].

The mixing sequences are generated by pseudo-random sequences. At the mixer, there will be one transmitter to be shifted in the baseband of each MWC physical channel. Thus, the more number of MWC physical channels there are, the more transmitters will be detected. In practice, the number of MWC physical channels cannot be high, due to the exponential increase cost of analog components such as mixer, lowpass filter and ADC. Consequently, a collapsing factor $q$ is introduced to overcome this problem [12]. Instead of $M$ physical channels, it can be considered as $q \times M$ collapsed channels. With $M$ channels, the sampling rate is $F_{s}$, thus with $q \times M$ channels, the sampling rate can be considered $F_{p}$, by $q=F_{s} / F_{p}$. This assumption can help to extend virtually the number of MWC channels.

\section{B. Number of active subbands estimation}

In Fig. 1, before reconstruction, the number of active subbands $s$ needs to be estimated. The output of the MWC $y_{i}[n]$ $(1 \leq i \leq M)$ has $L$ sub-Nyquist samples, it can be formed to a $M \times N$ matrix $\mathbf{y}$ output, or $q M \times L$ matrix $\mathbf{y}$ if collapsing factor $q$ is used.

The number of active subbands is estimated based on the singular values of matrix $\mathbf{y} \in \mathbb{P}^{M \times L}$, or the autocorrelation matrix eigenvalues. The active subbands $s$ is related to the variation of these values. Then, there are $s$ non-zero eigenvalues. In the simulation of this paper, the number of active subbands $s$ is estimated by two discriminant functions [26].

\section{Spectrum reconstruction}

The mixing sequences $p_{i}(t)$ is used to form a sensing matrix $(\mathbf{P})_{i l}=p_{i l}$ [10], where $p_{i l}=F_{p} \int_{-T_{p} / 2}^{T_{p} / 2} p_{i}(t) e^{-j 2 \pi l F_{p} t} d t$ are the Fourier coefficients obtained from $p_{i}(t)$. The Fourier transform of the input signal (multi-subband) in the $l^{\text {th }}$ subband is denoted $z_{l}[n]$ with $l \in[1, L]$. Thus, the MWC system equation with a MWC output and a sensing matrix is $\mathbf{y}=\mathbf{P z}$, with $\mathbf{P} \in \mathbb{C}^{q M \times L}$ [11] [12]. Fig. 2 shows an illustration of the system equation.

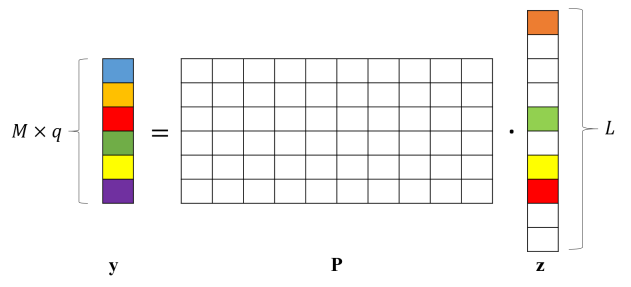

Fig. 2: Illustration of the system equation.

In Compressed Sensing, a main condition needed to be respected is $s \leq M q-1$, with $s$ the number of active subbands or the non-zero elements in $\mathbf{z}$. This condition is called sparsity because the matrix size of $\mathbf{y}(q \times M)$ has to be smaller than the size of input $\mathbf{z}$ ( $L$ elements) and there is a few number of $s$. The reconstruction step is to look for $\mathbf{z}$ from $\mathbf{y}$. In our simulation, a greedy algorithm Orthogonal Matching Pursuit (OMP) [27] is applied to reconstruct the input signal.

\section{MUltiPle POWER LEVELS SENSING AND RECONSTRUCTION}

For the rest of the paper, the MWC parameters are chosen as $M=4, L=96, q=7$ and $F_{N y q}=1 \mathrm{GHz}$. Each transmitter is a band-limited white noise and has the same bandwidth $B=2 F_{p} / 3=7 \mathrm{MHz}$. The central frequency of each transmitter has been randomly generated between $\left[\frac{B}{2}\right.$; $\left.\frac{F_{N y q}}{2}-\frac{B}{2}\right]$, i.e. [3.5;496.5] MHz. To respect the sparsity condition, the maximum number of symmetric transmitter must be equal to $N_{t_{\max }}=\left\lfloor\frac{M q-1}{4}\right\rfloor=6$ (by assuming that each transmitter can locate astride two channels of the equivalent model).

The spectrum is contaminated by a wideband additive Gaussian noise at different SNR. The SNR is defined into two 
categories, a SNR in all active subbands (or so-called global SNR in-band noticed IBSNR $_{\mathrm{G}}$ ) and a SNR in the whole Nyquist bandwidth noticed $\mathrm{SNR}_{\mathrm{Nyq}}$. Then, the relationship between the two categories of SNR can be seen as

$$
\begin{aligned}
\operatorname{IBSNR}_{\mathrm{G}} & =\mathrm{SNR}_{\mathrm{Nyq}}+10 \log \left(\frac{F_{N y q}}{2 \times N_{t} \times B}\right) \\
& =\mathrm{SNR}_{\mathrm{Nyq}}+10 \log \left(\frac{3 \times L}{4 \times N_{t}}\right)
\end{aligned}
$$

with $N_{t}$ the number of transmitters and $B=2 F_{p} / 3$. The number of transmitters is fixed to 6 .

The low $\mathrm{SNR}_{\mathrm{Nyq}}$ will make the active subbands to be aliased by the noise. It leads to the overestimation due to the unexpected high power in active subbands and difficult to determine the number of active subbands $s$ or number of transmitters $N_{t}$. Another factor that needs to be taken into account is the SNR in each transmitter bandwidth (not global SNR), this factor impacts directly to the reconstructions and in the next section, corresponding to each global In-Band SNR value $\mathrm{IBSNR}_{\mathrm{G}}$, the In-Band SNR in each transmitter bandwidth will be figured out.

Moreover, the correct reconstruction rate [28] (resp. false alarm rate) is estimated by $P_{c}=\frac{\%\left(B_{r} \cap B_{d}\right)}{\% B_{r}}$ (resp. $P_{f}=$ $\left.\frac{\%\left(\left(B_{d} \backslash B_{r}\right) \cap \bar{B}_{r}\right)}{1-\% B_{r}}\right)$, where $B_{r}$ the real active subbands from input signal and $B_{d}$ the detected active subbands. It should be noted that for each simulation scenario, 2000 trials have been tested to compute the reconstruction and false alarm rates.

To study the impact of transmitter power levels, with this configuration of the MWC, four scenarios are considered. In the first scenario, all six transmitters have the same power level. The second scenario, assume that one pair of transmitters has greatest power, then the second pair of transmitters has $75 \%$ level of the first pair, and the third pair has 50\% level of the first pair. Third scenario, we assume that the second pair has $50 \%$ level of the first pair, and the third pair has only $25 \%$ level of the first pair. In the last scenario, 6 transmitters have different power levels and reduce gradually Table I summarizes these scenarios.

\begin{tabular}{|c|c|c|c|c|c|c|}
\cline { 2 - 7 } \multicolumn{1}{c|}{} & $P_{1}$ & $P_{2}$ & $P_{3}$ & $P_{4}$ & $P_{5}$ & $P_{6}$ \\
\hline Scenario 1 & 1 & 1 & 1 & 1 & 1 & 1 \\
\hline Scenario 2 & 1 & 1 & 0.75 & 0.75 & 0.5 & 0.5 \\
\hline Scenario 3 & 1 & 1 & 0.5 & 0.5 & 0.25 & 0.25 \\
\hline Scenario 4 & 1 & 0.8 & 0.6 & 0.4 & 0.3 & 0.2 \\
\hline
\end{tabular}

TABLE I: Simulation scenarios and transmitters power levels.

\section{A. Reconstruction performances in function of Global In-Band Signal to Noise Ratio for all scenarios}

Firstly, the spectrum reconstruction will be tested at the same power level (scenario 1), then reduced the power levels of transmitters followed the scenarios in Table I, to obtain and compare the correct reconstruction and false alarm rates in function of $\mathrm{IBSNR}_{\mathrm{G}}$. Fig. 3 shows the correct reconstruction rate and false alarm rate for 6 transmitters of all scenarios. When reducing the power level, the correct reconstruction rate is reduced too. In scenario 2 , the $P_{c}$ in function of global SNR in-band is higher than the $P_{c}$ in scenario 3 and 4 , due to the fact that the proportion of useful power in an active subband is higher. In scenario 3 and 4 , the correct reconstruction rates and the false alarm rates are nearly the same, although the power levels in scenario 3 and 4 are different, the power proportion in all active subbands can be considered the same globally.
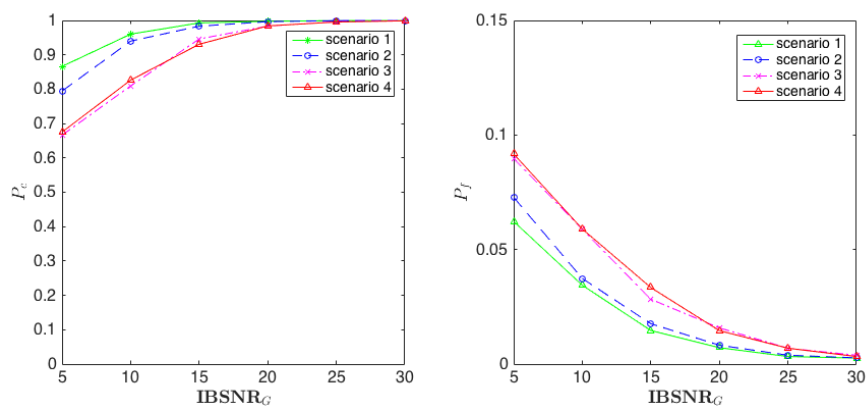

Fig. 3: Correct reconstruction rate and false alarm rate for all scenarios in function of $\operatorname{IBSNR}_{\mathrm{G}}$ (with $B=2 F_{p} / 3$ ).

It should be noted that from Fig. 3, the correct reconstruction rates start to decrease at $15 \mathrm{~dB}$ for scenario 1 and 2 , and at $20 \mathrm{~dB}$ for scenario 3 and 4 . Hence, these values can be considered as threshold values which ensure correct reconstruction. Due to the multiple thresholds for $\mathrm{IBSNR}_{\mathrm{G}}$, it is hard to determine which transmitter cannot be reconstructed, in next subsection, we propose a relationship to find an In-Band Signalto-Noise Ratio for each transmitter. This relationship helps to determine an unique IBSNR threshold for each transmitter and each scenario which ensures high reconstruction performances. Moreover, to verify the threshold, the bandwidth $B$ of each transmitter is reduced to $B=F_{p} / 3 \approx 3.47 \mathrm{MHz}$. The correct reconstruction and false alarm rates are shown in Fig 4. It can be seen that the $P_{c}$ at this bandwidth also starts to decrease at $15 \mathrm{~dB}$ for scenario 1 and 2 , and $20 \mathrm{~dB}$ for scenario 3 and 4 . This result helps to conclude that the proposed threshold can be used to make sure the correct reconstruction in these scenarios with an acceptable bandwidth.
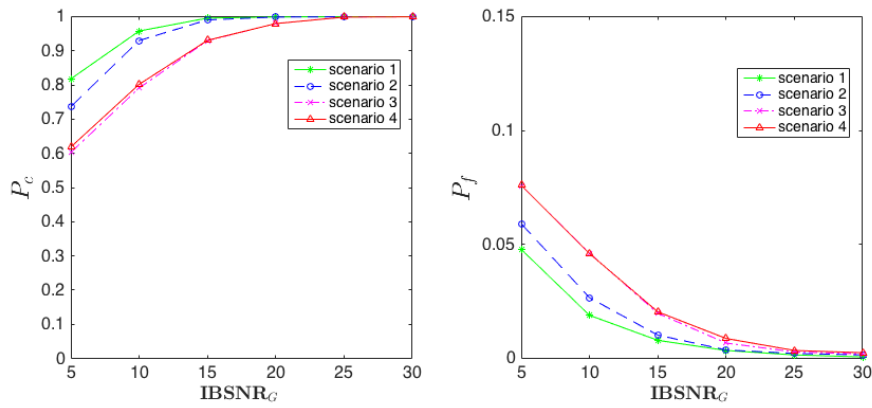

Fig. 4: Correct reconstruction rate and false alarm rate for all scenarios in function of $\operatorname{IBSNR}_{\mathrm{G}}$ (with $B=F_{p} / 3$ ).

Besides, an example of spectrum reconstruction of 6 identical power level transmitters is shown in Fig. 5 at $\mathrm{IBSNR}_{\mathrm{G}}=20$ 
$\mathrm{dB}$. It can be seen that at this noise level, all the transmitters can be correctly reconstructed with their level and no false alarm.
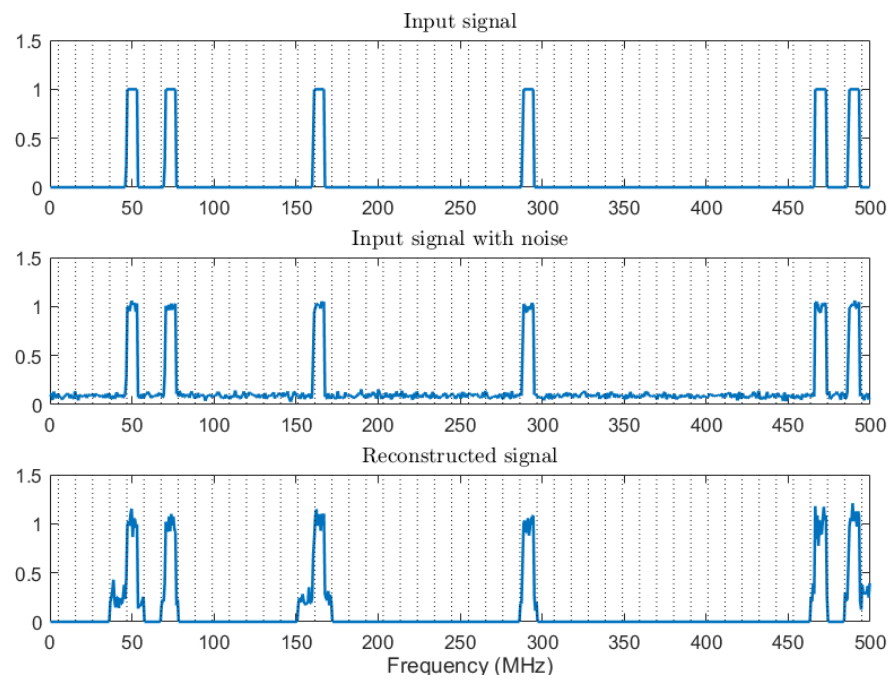

Fig. 5: Example of spectrum reconstruction of 6 identical power level transmitters at $\mathrm{IBSNR}_{\mathrm{G}}=20 \mathrm{~dB}$ (scenario 1).

From scenario 2 to 4 , we show the performance of reconstruction in function of the in-band Signal-to-Noise Ratio for each transmitter. It is determined by the ratio between signal levels which is linked to the in-band SNR of each transmitter taken separately. It is possible to compute the In-band Signalto-Noise Ratio for each transmitter $\mathrm{IBSNR}_{1 \mathrm{~T}}$ by:

$$
\begin{aligned}
\mathrm{IBSNR}_{1 \mathrm{~T}} & =\mathrm{SNR}_{\mathrm{Nyq}}^{1 \mathrm{~T}}+10 \log \left(\frac{F_{N y q}}{2 \times B}\right) \\
& =\mathrm{SNR}_{\mathrm{Nyq}}^{1 \mathrm{~T}}+10 \log \left(\frac{3 \times L}{4}\right)
\end{aligned}
$$

with $\mathrm{SNR}_{\mathrm{Nyq}}^{1 \mathrm{~T}}$ the SNR in the whole Nyquist bandwidth of the considered transmitter and $B=2 F_{p} / 3$.

\section{B. Correct reconstruction performance in function of In-Band Signal to Noise Ratio}

1) Scenario 2: In this scenario, the correct reconstruction rate is shown in Fig. 6, in function of the exact IBSNR ${ }_{1 T}$. It can be observed that at the same global in-band SNR, the low power level transmitters have to deal with higher noise than the higher power level, then leads to a bad reconstruction. It can be seen that the pair which has the greatest power level $\left(\left\{P_{1}, P_{2}\right\}=1\right)$ can achieve high performance even at low $\mathrm{IBSNR}_{\mathrm{G}}$. For the second pair $\left(\left\{P_{3}, P_{4}\right\}=0.75\right)$, the correct reconstruction rate is still high, more than $85 \%$ at $5 \mathrm{~dB}$ of IBSNR $_{1 \mathrm{~T}}$. Otherwise, the lowest level pair $\left(\left\{P_{5}, P_{6}\right\}=0.5\right)$ has low performance when IBSNR $_{1 \mathrm{~T}}$ is low (typically less than $5 \mathrm{~dB}$ ). It means that the reconstruction is not correct at this noise level. Overall, the correct reconstruction rate starts to decrease at $\mathrm{IBSNR}_{1 \mathrm{~T}}=15$ $\mathrm{dB}$, then this is a threshold proposed for this scenario to ensure the correct reconstruction for all transmitters.

Fig. 7 shows the correct reconstruction rate for this scenario with a reduction of bandwidth $\left(B=F_{p} / 3\right)$, it should be

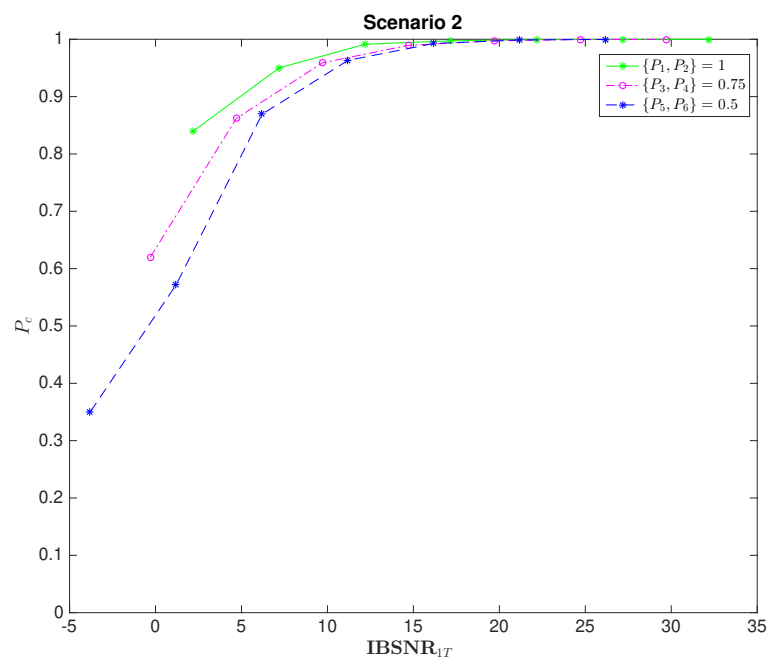

Fig. 6: Correct reconstruction rate of 6 transmitters with different power levels in function of $\mathrm{IBSNR}_{1 \mathrm{~T}}$ (scenario $2, B=2 F_{p} / 3$ ).

noted that $P_{c}$ starts to decrease at $\mathrm{IBSNR}_{1 \mathrm{~T}}=15 \mathrm{~dB}$ for all transmitters. This result verifies the proposed threshold for this scenario.

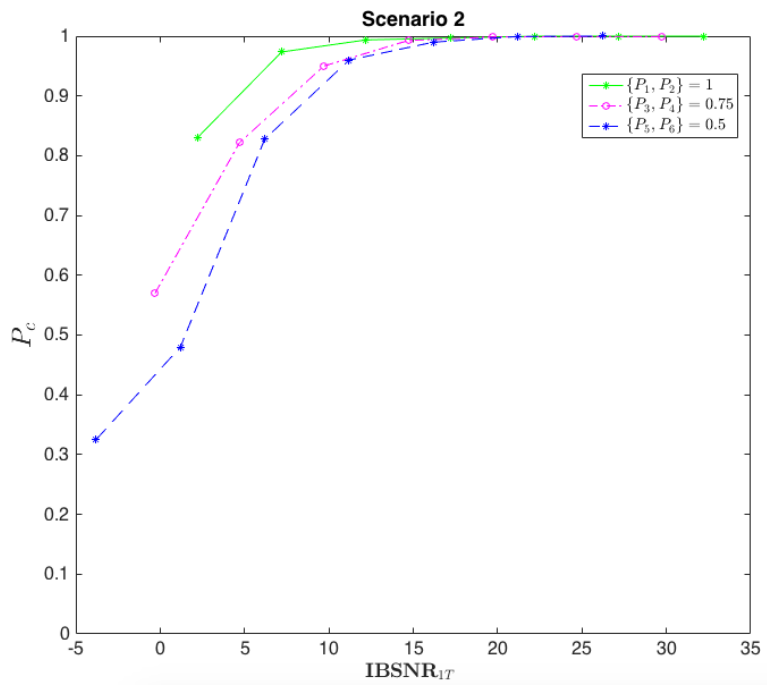

Fig. 7: Correct reconstruction rate of 6 transmitters with different power levels in function of $\operatorname{IBSNR}_{1 \mathrm{~T}}$ (scenario $2, B=F_{p} / 3$ ).

An example of spectrum reconstruction of this scenario at $\mathrm{IBSNR}_{\mathrm{G}}=20 \mathrm{~dB}$ is shown in Fig. 8. It can be seen that in this scenario and at this noise level, it is difficult to reconstruct the low power transmitters with their levels correctly. Moreover, it appears to be a false alarm and that makes confusion with low power transmitters.

Moreover, the spectrum reconstruction of this scenario at $\mathrm{IBSNR}_{\mathrm{G}}=5 \mathrm{~dB}$ is shown in Fig. 9. It is clear that at this noise level, the reconstruction is not absolutely correct, and false alarm spectra appear along the Nyquist bandwidth. For the blind spectrum detection application, this noise level $\left(\mathrm{IBSNR}_{\mathrm{G}}=5\right.$ 

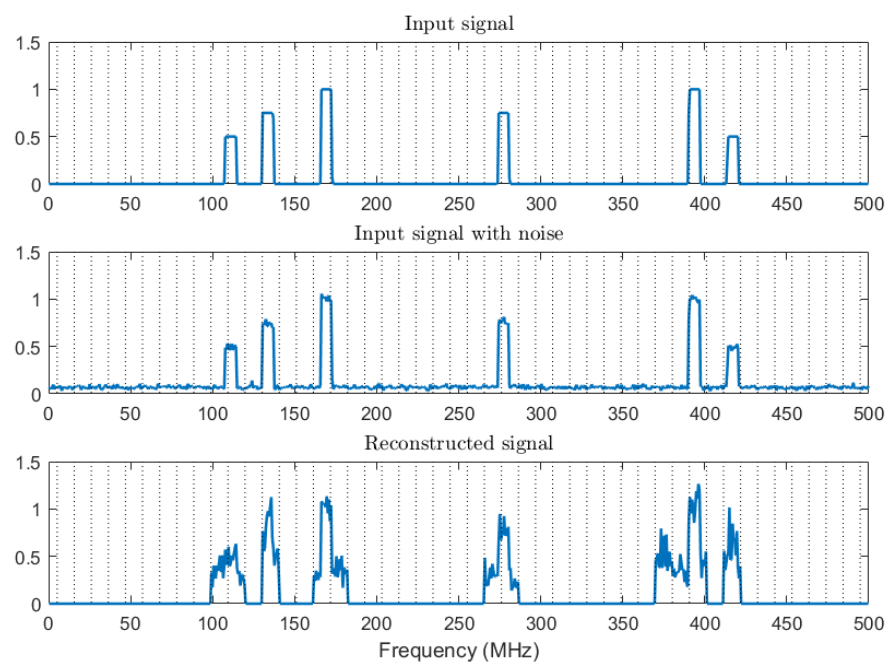

Fig. 8: Spectrum reconstruction of 6 transmitters with different power levels in scenario 2 at $\mathrm{IBSNR}_{\mathrm{G}}=20 \mathrm{~dB}$.

dB) makes it difficult to determine the useful transmitters.
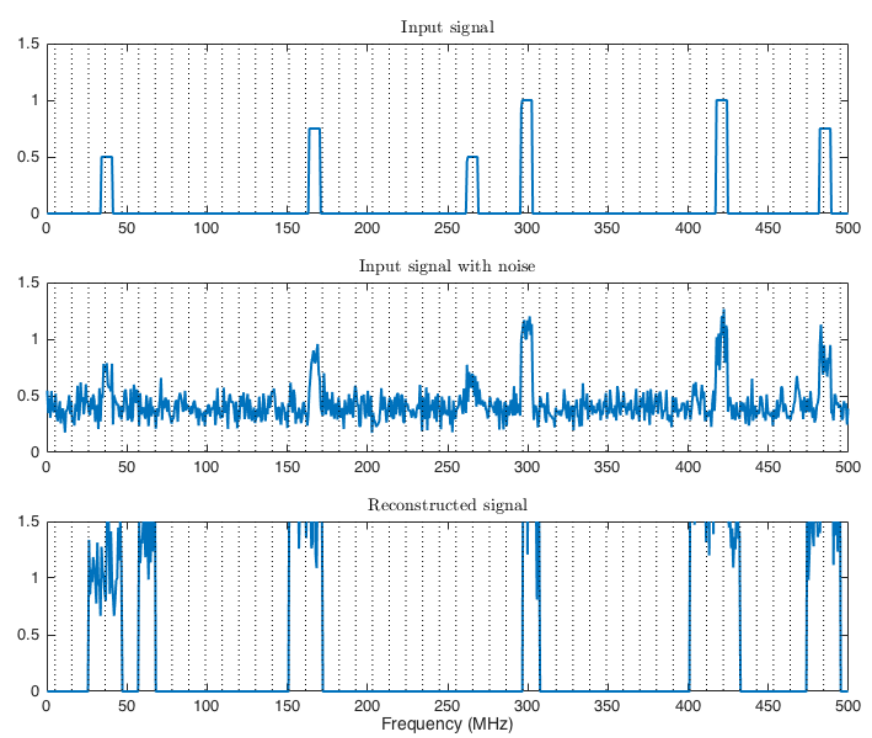

Fig. 9: Spectrum reconstruction of 6 transmitters with different power levels in scenario 2 at $\mathrm{IBSNR}_{\mathrm{G}}=5 \mathrm{~dB}$.

2) Scenario 3: In the third scenario, the power level of the second pair and third pair are continuously reduced $\left(\left\{P_{3}, P_{4}\right\}=\right.$ 0.5 and $\left.\left\{P_{5}, P_{6}\right\}=0.25\right)$. This scenario is to test the reconstruction performance of the MWC in case of very low power transmitters. The reconstruction rate is presented in Fig. 10 in function of $\mathrm{IBSNR}_{1 \mathrm{~T}}$. It is clear to see that the reconstruction rate of the third pair $\left(\left\{P_{5}, P_{6}\right\}=0.25\right)$ has low performance in the presence of noise (less than $6 \mathrm{~dB}$ ). Due to the power ratio of transmitters that is reduced in this scenario, the second pair can achieve higher performance than the previous scenario. Also, from Fig. 10, the reconstruction rate starts to decrease at $\mathrm{IBSNR}_{1 \mathrm{~T}}=15 \mathrm{~dB}$. This is considered as a threshold to ensure the correct reconstruction for all transmitters in this scenario.

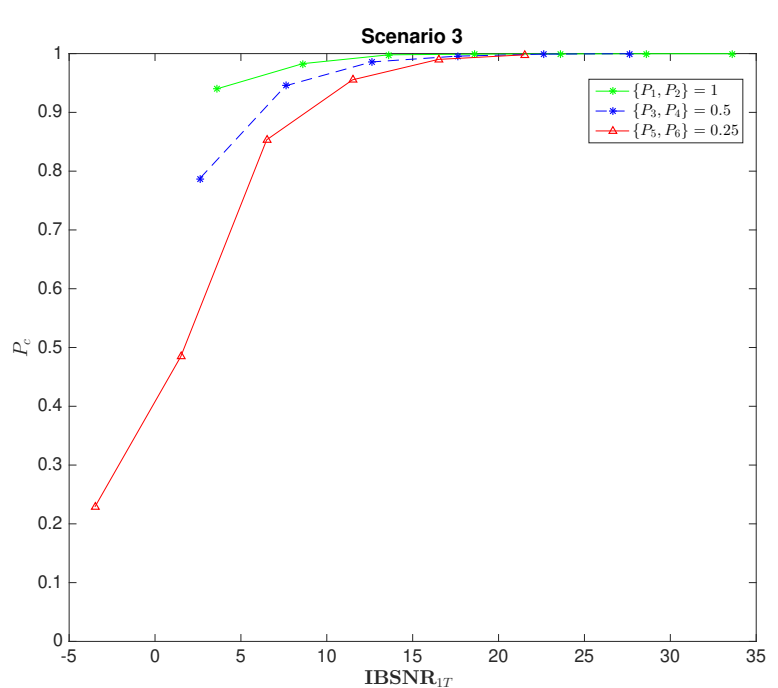

Fig. 10: Correct reconstruction rate of 6 transmitters with different power levels in function of $\operatorname{IBSNR}_{1 \mathrm{~T}}$ (scenario $3, B=2 f_{p} / 3$ ).

Moreover, Fig 11 illustrates the correct reconstruction rate for scenario 3 with the bandwidth $B=F_{p} / 3$. The performance of reconstruction can be ensured at $\geq 15 \mathrm{~dB}$ SNR of each transmitter, then the proposed threshold can be verified.

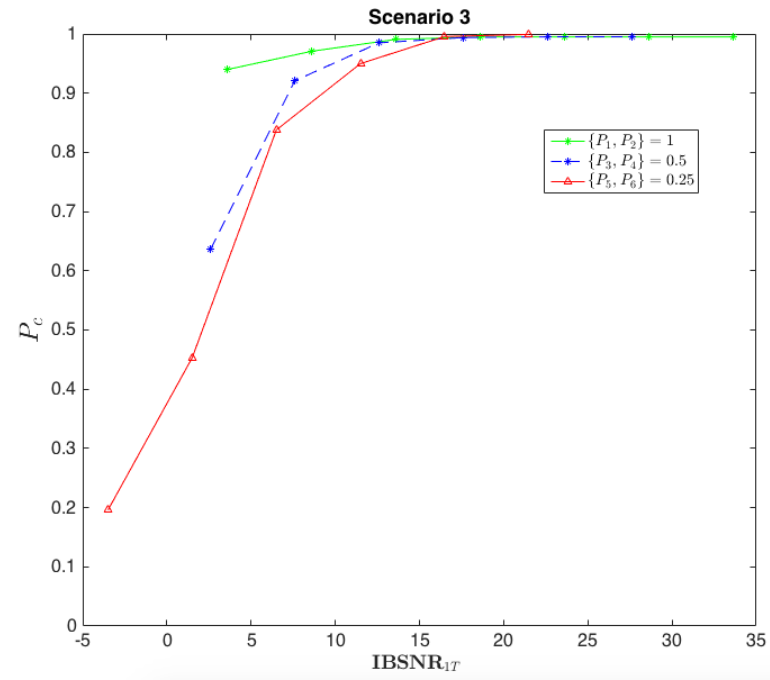

Fig. 11: Correct reconstruction rate of 6 transmitters with different power levels in function of $\operatorname{IBSNR}_{1 \mathrm{~T}}$ (scenario $3, B=F_{p} / 3$ ).

An example of spectrum reconstruction for the third scenario is presented in Fig. 12 at $\mathrm{IBSNR}_{\mathrm{G}}=20 \mathrm{~dB}$. It can be seen that in this scenario and at this noise level, the first pair and the second pair can be reconstructed correctly with their frequency location and their power level. The lowest power level is always difficult to reconstruct because the ratio between the lowest level with the maximum level is always low. Moreover, at this noise level, all the transmitters can be detected correctly and there is no false alarm. 

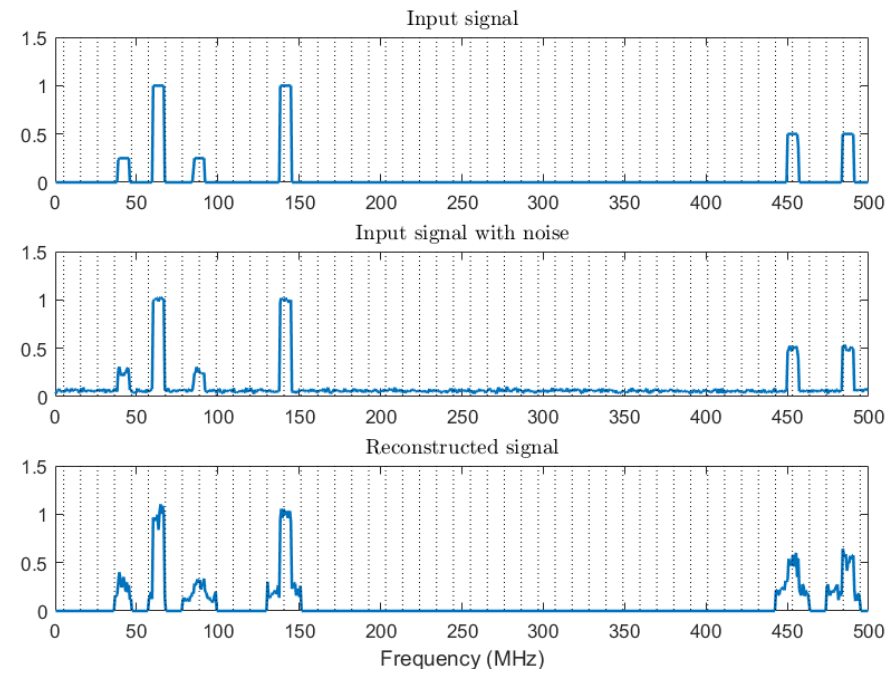

Fig. 12: Spectrum reconstruction of 6 transmitters with different power levels in scenario 3 at $\mathrm{IBSNR}_{\mathrm{G}}=20 \mathrm{~dB}$.

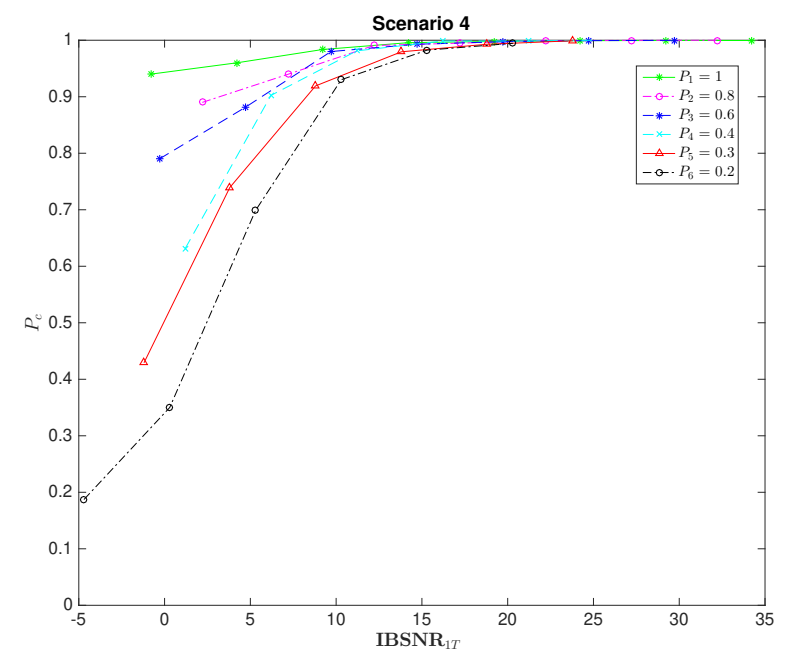

Fig. 13: Correct reconstruction rate of 6 transmitters with different power levels in function of $\mathrm{IBSNR}_{1 \mathrm{~T}}$ (scenario $4, B=2 F_{p} / 3$ ).

3) Scenario 4: This scenario is to study more levels, such as each transmitter has a different level to the others and is reduced gradually. The correct reconstruction rate and false alarm rate are shown in Fig. 13. It can be seen from this figure, the $P_{c}$ of the power level which is under $50 \%$ the maximum power (i.e. for $P_{4}=0.4, P_{5}=0.3$ and $P_{6}=0.2$ ) is low, even the global correct reconstruction rate, at low $\operatorname{IBSNR}_{\mathrm{G}}(5$ and 10 $\mathrm{dB})$. These values show that some transmitters have been lost at these power ratios and SNR levels. Moreover, from Fig. 3 the false alarm rate of this scenario is highest compared to the other scenarios. It means that the system cannot determine the frequency locations of the small power transmitters and then the wrong detection appears. Finally, a threshold proposed for this scenario is also at $15 \mathrm{~dB}$.

The correct reconstruction rate with the reduction of band-

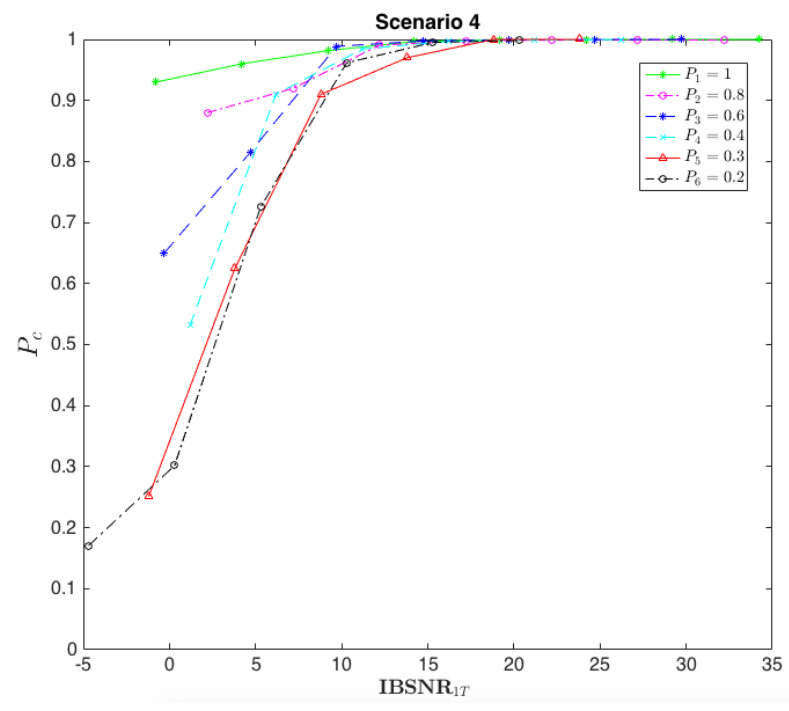

Fig. 14: Correct reconstruction rate of 6 transmitters with different power levels in function of $\operatorname{IBSNR}_{1 \mathrm{~T}}$ (scenario $4, B=F_{p} / 3$ ).

width $\left(B=F_{p} / 3\right)$ is shown in Fig. 14 , this also verifies that the proposed threshold is $\mathrm{IBSNR}_{1 \mathrm{~T}}=15 \mathrm{~dB}$ to ensure the correct reconstruction.

Besides, an example of spectrum reconstruction is shown in Fig. 15 at $\mathrm{IBSNR}_{\mathrm{G}}=20 \mathrm{~dB}$. At this noise level, all the transmitters can be detected correctly. The lowest power level transmitter $\left(P_{6}=0.2\right.$ in this example $)$ at $\mathrm{IBSNR}_{\mathrm{G}}=20$ $\mathrm{dB}$ is equivalent $\mathrm{IBSNR}_{1 \mathrm{~T}}=10.2 \mathrm{~dB}$ at its subbands. Then, the reconstruction rate of this level is nearly $90 \%$ and the false alarm rate is low, less than $2 \%$ (in Fig. 13). Hence, the reconstruction of MWC is correct in the frequency locations at this noise level and no false alarm. The power level is impacted by noise, however, the monitoring system cannot reconstruct exactly the transmitter power level, mainly some near value and low levels (for example, $P_{5}=0.3$ and $P_{6}=0.2$ ).

Based on our simulations, the threshold for IBSNR $1 \mathrm{~T}$, with an acceptable bandwidth, to ensure the correct reconstruction can be concluded is $15 \mathrm{~dB}$ for all cases.

\section{CONCLUSION}

This paper studies the impact of transmitters power levels on the reconstruction performance of the MWC system in terms of correct reconstruction and false alarms rates. It is clear that the noise power has a negative impact on the reconstruction performance, by adding false alarm and by introducing miss detection, especially for transmitters with low power levels. Based on our simulations, reconstruction performances depend on the In-Band Signal to Noise ratio (IBSNR) of each transmitter and a threshold value of $\operatorname{IBSNR}_{1 \mathrm{~T}}=15 \mathrm{~dB}$ has been proposed to ensure high reconstruction performances.

\section{ACKNOWLEDGMENT}

This research is supported by the IBNM (Brest Institute of Computer Science and Mathematics) CyberIoT Chair of Excellence at the University of Brest. 

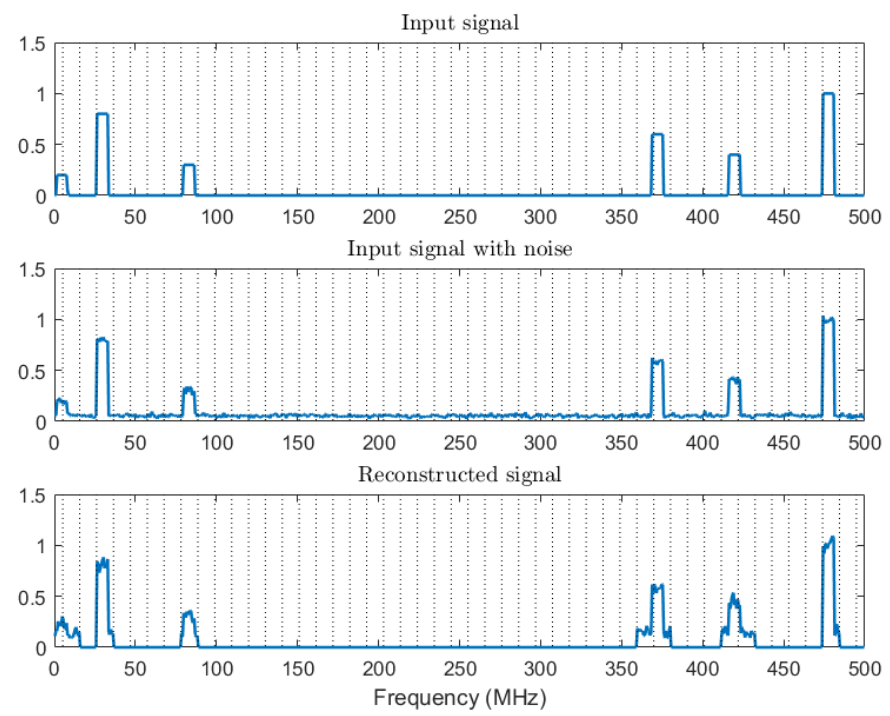

Fig. 15: Spectrum reconstruction of 6 transmitters with different power levels in scenario 4 at $\mathrm{IBSNR}_{\mathrm{G}}=20 \mathrm{~dB}$.

\section{REFERENCES}

[1] J. Mitola, "Cognitive radio architecture evolution," Proc. IEEE, vol. 97, no. 4, pp. 626-641, 2009.

[2] Ž. Tabaković, "A survey of cognitive radio systems," Post Electron. Commun. Agency, vol. 13, 2011.

[3] R. Tandra and A. Sahai, "SNR walls for signal detection," IEEE J. Sel. Top. in Sig. Process., vol. 2, no. 1, pp. 4-17, 2008.

[4] Z. Chen, F. Gao, X. Zhang, J. C. Li, and M. Lei, "Sensing and power allocation for cognitive radio with multiple primary transmit powers," IEEE Wireless Communications Letters, vol. 2, no. 3, pp. 319-322, 2013.

[5] F. Gao, J. Li, T. Jiang, and W. Chen, "Sensing and recognition when primary user has multiple transmit power levels," IEEE Transactions on signal Processing, vol. 63, no. 10, pp. 2704-2717, 2015.

[6] J. N. Laska, S. Kirolos, M. F. Duarte, T. S. Ragheb, R. G. Baraniuk, and Y. Massoud, "Theory and implementation of an analog-to-information converter using random demodulation," in IEEE Int. Symp. on Circuits and Syst., pp. 1959-1962, IEEE, 2007.

[7] J. A. Tropp, J. N. Laska, M. F. Duarte, J. K. Romberg, and R. G. Baraniuk, "Beyond nyquist: Efficient sampling of sparse bandlimited signals," IEEE Trans. Inf. Theo., vol. 56, no. 1, pp. 520-544, 2010.

[8] R. Venkataramani and Y. Bresler, "Perfect reconstruction formulas and bounds on aliasing error in sub-Nyquist nonuniform sampling of multiband signals," IEEE Trans. Inf. Theory, vol. 46, pp. 2173-2183, Sept, 2000.

[9] M. Mishali and Y. C. Eldar, "Blind multiband signal reconstruction: Compressed sensing for analog signals," IEEE Trans. on Sig. Process., vol. 57, no. 3, pp. 993-1009, 2009.
[10] M. Mishali and Y. C. Eldar, "From theory to practice: Sub-nyquist sampling of sparse wideband analog signals," IEEE J. Sel. Top. Sig. Process., vol. 4, no. 2, pp. 375-391, 2010.

[11] M. Mishali, Y. C. Eldar, O. Dounaevsky, and E. Shoshan, "Xampling Analog to digital at sub-Nyquist rates," IET Cir., Devices and Syst., vol. 5, pp. 8-20, Jan. 2011.

[12] M. Mishali, Y. C. Eldar, and A. J. Elron, "Xampling: Signal acquisition and processing in union of subspaces," IEEE Trans. Sig. Process., vol. 59, no. 10, pp. 4719-4734, 2011.

[13] M. A. Lexa, M. E. Davies, J. S. Thompson, and J. Nikolic, "Compressive power spectral density estimation," in Int. Conf. Acoust., Speech and Sig. Process., pp. 3884-3887, IEEE, 2011.

[14] D. L. Donoho, "Compressed Sensing," IEEE Trans. Inform. Theo., vol. 52, no. 4, pp. 1289-1306, 2006.

[15] E. J. Candès, J. Romberg, and T. Tao, "Robust uncertainty principles: Exact signal reconstruction from highly incomplete frequency information," IEEE Trans. Inform. Theo., vol. 52, no. 2, pp. 489-509, 2006.

[16] E. Israeli, S. Tsiper, D. Cohen, E. Shoshan, R. Hilgendorf, A. Reysenson, and Y. C. Eldar, "Hardware calibration of the modulated wideband converter," in IEEE Glob, Commun. Conf., pp. 948-953, IEEE, 2014.

[17] D. Cohen, S. Tsiper, and Y. C. Eldar, "Analog to Digital Cognitive Radio: Sampling, Detection, and Hardware," IEEE Sig. Process. Mag., vol. 35, no. 1, pp. 137-166, 2018.

[18] P. De and U. Satija, "Sparse representation for blind spectrum sensing in cognitive radio: a compressed sensing approach," Circuits, Systems, and Signal Processing, vol. 35, no. 12, pp. 4413-4444, 2016.

[19] C. B. A. Wael, N. Armi, B. P. Rohman, and T. Miftahushudur, "Performance analysis of sub-nyquist sampling for wideband spectrum sensing in cognitive radio," in 2016 International Conference on Radar, Antenna, Microwave, Electronics, and Telecommunications (ICRAMET), pp. 152156, IEEE, 2016.

[20] Z. SONG, Y. GAO, and R. TAFAZOLLI, "A survey on spectrum sensing and learning technologies for 6g," IEICE Transactions on Communications, p. 2020DSI0002, 2021.

[21] D. J. K. Adams, A practical implementation of the Modulated Wideband Converter Compressive Sensing receiver architecture. $\mathrm{PhD}$ thesis, Stanford University, 2016.

[22] A. Sahai, N. Hoven, and R. Tandra, "Some fundamental limits on cognitive radio," in Allerton Conf. Commun., Contr., and Computing, pp. 1662-1671, Monticello, Illinois, 2004.

[23] I. Develi et al., "Spectrum sensing in cognitive radio networks: threshold optimization and analysis," EURASIP Journal on Wireless Communications and Networking, vol. 2020, no. 1, pp. 1-19, 2020.

[24] R. Gold, "Optimal binary sequences for spread spectrum multiplexing (corresp.)," IEEE Trans. Inf. Theory, vol. 13, no. 4, pp. 619-621, 1967.

[25] T. Kasami, "Weight distribution formula for some class of cyclic codes," Coordinated Science Laboratory Report no. R-285, 1966.

[26] E. Radoi and A. Quinquis, "A New Method for Estimating the Number of Harmonic Components in Noise with Application in High Resolution Radar," J. on Applied Sig. Process., pp. 1177-1188, 2004.

[27] T. T. Cai and L. Wang, "Orthogonal Matching Pursuit for sparse signal recovery with noise," IEEE Trans. on Inf. Theory, vol. 57, no. 7, pp. 46804688, 2011.

[28] L. L. Nguyen, R. Gautier, A. Fiche, G. Burel, and E. Radoi, "Digital compensation of lowpass filters imperfection in the Modulated Wideband Converter Compressed Sensing scheme for radio frequency monitoring," Sig. Process., vol. 152, pp. 292-310, 2018. 